\title{
Diacronie
}

Studi di Storia Contemporanea

$\mathrm{N}^{\circ} 36,4 \mid 2018$

Viaggi e turismo nell'Europa del Novecento

\section{Gerardo Padulo, L'ingrata progenie. Grande guerra, Massoneria e origini del Fascismo (1914-1923)}

\section{Edoardo Grassia}

\section{Q OpenEdition}

\section{Edizione digitale}

URL: https://journals.openedition.org/diacronie/10471

DOI: 10.4000/diacronie. 10471

ISSN: 2038-0925

Editore

Association culturelle Diacronie

Notizia bibliografica digitale

Edoardo Grassia, «Gerardo Padulo, L'ingrata progenie. Grande guerra, Massoneria e origini del Fascismo (1974-1923)», Diacronie [Online], №36, 4 | 2018, documento 15, online dal 29 décembre 2018,

consultato il 17 février 2022. URL: http://journals.openedition.org/diacronie/10471 ; DOI: https://

doi.org/10.4000/diacronie.10471 


\title{
Diacronie
}

Studi di Storia Contemporanea

$36,4 / 2018$

Viaggi e turismo nell'Europa del Novecento

\section{RECENSIONE: Gerardo PADULO, L'ingrata progenie. Grande guerra, Massoneria e origini del Fascismo (1914-1923), Siena, Nuova Immagine Editrice, 2018, 200 pp.}

\author{
A cura di Edoardo GRASSIA
}

Per citare questo articolo:

GRASSIA, Edoardo, «RECENSIONE: Gerardo PADULO, L'ingrata progenie. Grande guerra, Massoneria e origini del Fascismo (1914-1923), Siena, Nuova Immagine Editrice, 2018, 200 pp.», Diacronie. Studi di Storia Contemporanea : Viaggi e turismo nell'Europa del Novecento, 36, 4/2018, 29/12/2018,

URL: < http://www.studistorici.com/2018/12/29/grassia_numero_36/ >

Diacronie Studi di Storia Contemporanea $\rightarrow$ http://www.diacronie.it

Rivista storica online. Uscita trimestrale.

redazione.diacronie@hotmail.it

Comitato di direzione: Naor Ben-Yehoyada - João Fábio Bertonha - Christopher Denis-Delacour - Maximiliano Fuentes Codera Anders Granås Kjøstvedt - John Paul Newman - Deborah Paci - Niccolò Pianciola - Spyridon Ploumidis - Wilko Graf Von Hardenberg

Comitato di redazione: Jacopo Bassi - Luca Bufarale - Gianluca Canè - Luca G. Manenti - Fausto Pietrancosta - Alessandro Salvador - Matteo Tomasoni - Luca Zuccolo 


\title{
15/ RECENSIONE: Gerardo PADULO, L'ingrata progenie. Grande guerra, Massoneria e origini del Fascismo (1914-1923), Siena, Nuova Immagine Editrice, 2018, $200 \mathrm{pp}$.
}

\author{
A cura di Edoardo GRASSIA
}

L'intenso e mutevole rapporto tra la massoneria e il fascismo, la ricostruzione e l'analisi delle loro relazioni, delle attività comuni e delle loro contrapposizioni, con le variabili di "quale massoneria", in funzione della loggia di cui si vuol discutere, e di "quale fascismo", in relazione al diverso volto che il regime ha progressivamente mostrato, è stato un argomento che ha interessato studiosi e ricercatori delle diverse discipline storiche e sociali, soprattutto nell'ultimo ventennio. Limitandoci alla saggistica storica, non passano inosservati i lavori di Mola ${ }^{1}$, di Isastia ${ }^{2}$, di Conti ${ }^{3}$ e di Venzi ${ }^{4}$, solo per citarne alcuni in rigoroso ordine temporale. All'argomento sono dedicate anche alcune ricerche tra le quali indichiamo quelle di Livi5 e Melfa ${ }^{6}$.

L'interesse, accademico e non, per questo argomento, per cause che non andremo ad analizzare, dopo gli studi iniziali sul finire degli anni Settanta del Novecento7, sembrerebbe essere sbocciato nel primo decennio del Duemila.

Nel 2018 Gerardo Padulo, per i tipi della Nuova Immagine Editrice, ha voluto tornare sulla questione. Padulo è uno storico, dottore di ricerca, dal lungo curriculum. Tra le varie attività, è stato borsista dell'Istituto Italiano di Studi Storici di Napoli e della Fondazione Einaudi di Torino. Sempre a Torino ha poi lavorato presso il Centro Studi Gobetti ed è stato chiamato quale consulente parlamentare per la Commissione Stragi e per la Commissione Mitrokhin. Ha svolto un

\footnotetext{
${ }^{1}$ MOLA, Aldo Alessandro, Storia della Massoneria italiana dalle origini ai giorni nostri, Milano, Bompiani, 2001.

${ }^{2}$ ISTASTIA, Anna Maria, Massoneria e fascismo. La repressione degli anni Venti, Firenze, Libreria Chiari, 2003.

${ }^{3}$ CONTI, Fulvio, Storia della massoneria italiana: dal risorgimento al fascismo, Bologna, Il Mulino, 2006.

${ }^{4}$ VENZI, Fabio, Massoneria e fascismo: dall'intesa cordiale alla distruzione delle logge: come nasce una "guerra di religione" 1921-1925, Roma, Castelvecchi, 2008.

${ }^{5}$ LIVI, Angelo, Massoneria a fascismo, Foggia, Bastogi, 2000.

${ }^{6}$ MELFA, Valentina Marica, Massoneria e fascismo. Dall'interventismo alla lotta partigiana, Catania, Bonanno, 2010.

${ }^{7}$ MOLA, Aldo Alessandro, op. cit.; VANNONI, Gianni, Massoneria, fascismo e chiesa cattolica, Bari, Laterza, 1979.
} 
analogo incarico di consulenza storica anche per diverse procure italiane. I suoi ambiti di ricerca hanno spaziato dalle questioni nittiane alla fascistizzazione della stampa alla massoneria ${ }^{8}$.

Il nuovo studio proposto ha catturato la nostra iniziale attenzione per il suo titolo: L'ingrata progenie. Grande Guerra, la massoneria e le origini del fascismo che, chiaramente indicante l'argomento, ci ha fatto riflettere sulle possibilità di fornire nuovi apporti documentali e storici sulla storia della massoneria in Italia. Anticipiamo che Padulo, da subito, ha annullato tale aspettativa iniziale. L'impressione di trovarci tra le mani una "storia della massoneria" è subito caduta.

L'autore, con uno stile colloquiale, sembra porsi davanti al suo interlocutore immaginario per raccontare, con qualche interruzione e con l'apertura di alcune parentesi necessarie per una spiegazione/comprensione più allargata di alcuni singoli avvenimenti, la sua esperienza di ricerca. La lettura è sicuramente piacevole, seppur presenta, talvolta, ampi riferimenti diretti, con la trascrizione integrale della documentazione analizzata che appesantisce il ruolo del lettore - in un testo storiografico, chiaramente, la citazione diretta delle fonti ha sempre una sua ragion d'essere! - ma che, al tempo stesso, può stimolarlo con la curiosità e con una sua interpretazione soggettiva. In alcune circostanze si ha l'impressione di essere parte attiva nelle argomentazioni esposte, come quando Padulo indica diverse possibilità di lettura del medesimo avvenimento e diversi interrogativi, per giungere alla più logica soluzione, per la quale l'autore fornisce, comunque, la spiegazione delle sue deduzioni. Queste - e l'autore con onestà lo scrive chiaramente - emergono anche quando le congetture legate a importanti avvenimenti non sono sufficientemente supportate dalle fonti documentali, perché ad oggi non reperibili, ma che non si dispera di rintracciare.

Lo studio, oltre a darci l'impressione di non voler essere una "storia della massoneria" non risulta neanche "semplicemente" inquadrabile in un generico "massoneria e fascismo", che primeggia nei titoli delle ricerche di settore. Il testo inverte l'ordine di questi termini e sviluppa un'analisi sul "fascismo massonico", ovvero sul pre-fascismo coltivato nell'associazione massonica e su come i caratteri, il pensiero e l'ideologia iniziale del primo fossero già insiti, seppur privi di un nome identificativo, nella seconda, ovvero nei sui iscritti. Questa base porta all'analisi del

\footnotetext{
${ }^{8}$ Tra le principali produzioni, ricordiamo: PADULO, Gerardo, I rapporti tra Nitti e Giolitti, in Piero Gobetti e la Francia: atti del colloquio italo francese, 25-27 febbraio 1983, Milano, Angeli, 1983, pp. 145-195; ID., «Sui rapporti tra gli industriali del il governo Nitti (23 giugno 1919-9 giugno 1920)», in Nuova Rivista Storica, LX, 1976, pp. 591-603; ID., «Il partito nazionale fascista a Torino: una nuova fonte», in Mezzosecolo: materiali di ricerca storica, 8, 1989, pp. 89-91; ID., «Un interrogatorio su G. Amendola del 1924», in Prospettive settanta, 4, 1979, pp. 538-540; ID., I finanziatori del fascismo, Siena, Nuova immagine, 2010. Per lo specifico tema "massoneria e fascismo" cfr.: ID., «Palazzo Giustiniani a/e piazza San Sepolcro», in Mezzosecolo: materiali di ricerca storica, 6, 1985/1986, pp. 123-145; ID., "Contributo alla storia della massoneria da Giolitti a Mussolini», in Annali dell'Istituto italiano per gli studi storici, VIII, 1983/1984, pp. 220-347; ID., «Bonaldo Stringhet, massone senza matricola e senza numero d'ordine», in Le Carte e la Storia, 1/2016, pp. 174-178.
} 
rapporto di filiazione, come da titolo del testo, e spiega l'immediato appoggio che la massoneria fornì al primissimo, allora sconosciuto e inesplorato, movimento fascista.

Con la specificazione che si è rivolto alla sola massoneria della loggia di Palazzo Giustiniani 9 , la ricerca ruota attorno a tre momenti delicati e fondamentali per le conseguenze dirette e indirette prodotte: la fondazione de «Il Popolo d'Italia», del 15 novembre 1914, allora recante il sottotitolo «quotidiano socialista»; l'adunata a piazza San Sepolcro, del 23 marzo 1919, che decretò la nascita dei fasci di combattimento; la marcia su Roma, "opera buffa" secondo l'accezione di Salvemini ${ }^{10}$ o, ancora, "goffa kermesse" secondo Repaci"1, del 28 ottobre 1922. Tre episodi fondamentali per i successivi sviluppi storici nazionali e internazionali dove, a parere di Gerardo Padulo, la massoneria ebbe il ruolo di alma mater ${ }^{12}$. L'autore, in particolare e secondo noi a buon ragione, non fornisce eccessivi approfondimenti su questi avvenimenti, ma li analizza nelle loro fasi gestazionali e nei momenti immediatamente contemporanei al loro verificarsi, osservando e analizzando il decennio 1914-1923.

Proprio a partire dal periodo considerato, dai soggetti coinvolti e dai tre avvenimenti su cui si concentra, non nascondiamo la nostra difficoltà nel riporre il testo in uno specifico ambito tematico. Prima di collocarlo istintivamente nel suo "forse naturale" scaffale con i saggi afferenti al soggetto "massoneria", il primo spazio in cui ci sentiremmo di riporlo è assieme agli studi sulla Grande Guerra. In questi anni di "centenario", tra nuove pubblicazioni e rivisitazioni di testi più datati, il primo conflitto mondiale è stato esaminato da moltissimi studiosi, e altrettanto improvvisati e improbabili autori, e l'argomento ha potuto colmare gli scaffali delle librerie. L'ingrata progenie, riteniamo, si discosta dalla maggior parte di quella produzione storica scientifica e, pur non entrando mai in trincea, merita l'attenzione di chi vuole conoscere e approfondire le cause che portarono l'Italia alla dichiarazione di guerra, individuate da Padulo in un governo privo di una forte base parlamentare, nella caduta di Salandra per mano delle "massoneria internazionale" $13 \mathrm{e}$ dal conseguente avvicendamento alla presidenza del consiglio di due massoni, Boselli e Orlando's4. La Grande Guerra italiana, secondo le argomentazioni esposte

\footnotetext{
${ }^{9}$ Il 24 giugno 1908 vi fu una scissione nella massoneria italiana determinata dal diverso indirizzo dei massoni nel rapporto da tenere con la Chiesa. Le divergenze culminarono con la decisione di appoggiare o meno la proposta di legge di Leonida Bissolati sull'abolizione dell'insegnamento della religione nelle scuole elementari, e determinò la separazione tra Loggia di Palazzo Giustiniani, cui fa riferimento Gerardo Padulo, (cfr. PADULO, Gerardo, L'ingrata progenie. Grande guerra, Massoneria e origini del Fascismo (1914-1923), Siena, Nuova Immagine Editrice, 2018, p. 7), e Loggia di Piazza del Gesù.

${ }^{10} \mathrm{Cfr}$. SALVEMINI, Gaetano, Carteggio 1921-1926, a cura di Enzo TAGLIACOZZO, Roma-Bari, Laterza, 1985.

${ }^{11} \mathrm{Cfr}$. REPACI, Antonino, La marcia su Roma, Milano, Rizzoli, 1972.

${ }^{12}$ PADULO, Gerardo, L'ingrata progenie, cit., p. 13.

${ }^{13}$ Cfr. FIORI, Antonio, «Crisi e caduta del governo Salandra», in Rassegna storica del Risorgimento, 4/2003, pp. 537-574.

${ }^{14}$ PADULO, Gerardo, L'ingrata progenie, cit., p. 36.
} 
dall'autore, sarebbe, quindi, «in una certa misura»15 un conflitto voluto, guidato e gestito dalla massoneria. Abituati ai giri di valzer e alle analisi geopolitica, alla questione irredentista, al completamento del Risorgimento italiano e alla frattura interventista-neutralista, solo per indicare alcuni percorsi particolarmente fruttuosi, con l'analisi proposta da Padulo ci troviamo di fronte ad un nuovo tassello interpretativo: il ruolo - sia chiaro, entro certi limiti - giocato dalla massoneria nell'entrata in guerra dell'Italia. Il testo, in realtà, indica questo tema ma non lo approfondisce, lasciando ancora margini di studio che possano "quantificare" il peso della logga massonica nella scelta di campo e nella dichiarazione di guerra italiana, mettendola anche in relazione con altri elementi - i "poteri forti" come li potremmo chiamare, dall'industria alle banche - o con eventuali correnti massoniche neutraliste, ad esempio. Questo non toglie, chiaramente, l'importante contributo di questo studio e l'acclarata necessità che lo storico prenda in considerazione ed evidenzi tale percorso di ricerca. Qui risiede il pregio del volume di Padulo che, tra le moltissime pubblicazioni sulla Grande Guerra del nostro periodo, è stato tra i pochi a percorrerla.

Il testo, però, merita ulteriori collocazioni.

Considerato l'apporto fornito agli studi sul fascismo e alla sua genesi, L'ingrata progenie potrebbe essere riposto anche tra i testi che si occupano della nascita e dello sviluppo del regime totalitario in Italia, a partire dalla pubblicazione del quotidiano "socialista", ma soprattutto interventista, «Il Popolo d'Italia». In questo caso Padulo ne illustra il legame con la massoneria attraverso le basi finanziarie fornite direttamente da iscritti alla confraternita o indirettamente da società anonime comunque amministrate da membri della massoneria. Questo, nella complessiva struttura del testo, ci appare un momento portante dell'intero studio: la massoneria fa proprie le istanze dell'interventismo e ne rende possibile una voce attraverso il quotidiano diretto da Mussolini. Questo rapporto, nato dagli interessi comuni che avvicinarono le parti nel 1914, continuerà, saldandosi ancor di più, al termine del conflitto e si concretizzerà nella fondamentale presenza di iscritti alla massoneria nelle riunioni preparatorie e nella fondazione dei fasci da combattimento come movimento politico, avvenuta cinque anni dopo. Tale evoluzione, argomentata dalla lettura incrociata delle diverse fonti vagliate, porta Gerardo Padulo ad un importante suggerimento: spostare, anticipandoli, i termini di studio della genesi del movimento fascista. Se, da Salvemini ${ }^{16}$ a Tasca ${ }^{17}$ a De Felice ${ }^{18}$, siamo stati abituati ad assumere il 4 novembre 1918 quale data simbolo per comprendere il processo che ha portato Mussolini al

\footnotetext{
${ }^{15}$ Ibidem.

${ }^{16}$ Cfr. SALVEMINI, Gaetano, Le origini del fascismo. Lezioni di Harvard, a cura di Roberto VIVARELLI, Milano, Feltrinelli, 2015.

${ }^{17}$ Cfr. TASCA, Angelo, Nascita e avvento del fascismo: l'Italia dal 1918 al 1922, Firenze, La Nuova Italia, 1963.

${ }^{18} \mathrm{Cfr}$. DE FELICE, Renzo, Mussolini il fascista. La conquista del potere (1921-1925), Torino, Einaudi, 2005.
} 
governo, alla luce delle argomentazioni di Padulo ci sentiamo di condividere la sua proposta di anticiparne l'analisi agli avvenimenti del 1914.

Finita la guerra, tutti ebbero la necessità di rivolgersi al futuro e quello, per la massoneria e per qualsiasi movimento, poteva risiedere solo nella politica di massa. Continuando a far leva sul binomio patriottismo-antipatriottismo dell'anteguerra, si giunse, con passi veloci, in piazza San Sepolcro. É il secondo degli avvenimenti su cui si concentra il testo, ma come traguardo finale di un processo ricco di presenze massoniche, come uomini e come luoghi che resero possibile la riunione del 23 marzo 1919, indicata quale «epifania massonica» ${ }^{19}$. Non fu solo una manifestazione in forma visibile. Dopo quell'adunata di Milano, iniziarono altre moltissime azioni che sfociarono negli episodi di violenza e guerra civile, con l'intesa tra le prime squadre fasciste e l'Esercito, con la distribuzione di moschetti e munizioni e la diretta partecipazione dei militari in servizio. Anche questa intesa, come argomenta l'autore, sembrerebbe essere passata attraverso la massoneria, per "rapporti istituzionali" o per adesione massonica dei personaggi coinvolti a tutti i livelli: dall'Ufficio Informazioni dello Stato Maggiore Esercito agli Uffici Stampa e Propaganda dei comandi territoriali con quest'ultimi che, già attivi durante la Grande Guerra, ebbero un ruolo non secondario nelle violenze fasciste.

Ultimo elemento su cui si concentra la ricerca, "l'opera buffa", chiude il testo. La marcia su Roma, al di là della presenza massonica, rappresenta la conclusione di un processo iniziato nel 1914. Da quel 28 ottobre 1922, prese avvio un secondo processo, distinto dal primo, che porterà alla frattura incolmabile tra il movimento e poi regime del duce e l'associazionismo massonico ${ }^{20}$.

L'ingrata progenie è un testo che, qualsiasi sia la collocazione che gli si voglia dare, fornisce un sicuro apporto agli argomenti trattati. L'opera, sia complessivamente intesa che nelle sue “sezioni” interne, dal «Il Popolo d'Italia» alla marcia su Roma, si fa sicuramente apprezzare per la pertinenza, l'interesse e l'originalità di quanto illustrato, con la sola sentita necessità di poter leggere, in un successivo volume, un suo completamento. Se le argomentazioni di Padulo supportano sicuramente le sue conclusioni, si avverte, però, il desiderio di conoscere anche gli aspetti della "massoneria dissidente", per comprendere se e quanto essa abbia inciso sulla progenie, ovvero le eventuali potenzialità da una "massoneria liberale" 0 , in maniera ancor più estrema, la presenza e la reale forza di una massoneria o di singoli massoni vicini alle nascenti forze della sinistra politica e sociale. Questo, in particolare, ci permetterà di meglio confutare l'affermazione, un po' estrema e forse un po' provocatoria, secondo cui la società di fratellanza

\footnotetext{
${ }^{19}$ PADULO, Gerardo, L'ingrata progenie, cit., p. 127.

${ }^{20}$ La frattura tra massoneria e fascismo fu immediata e profonda tanto che Mussolini volle chiudere la questione alla quarta riunione del Gran Consiglio del Fascismo, il 15 febbraio 1923. In quell'occasione fu dichiarata l'incompatibilità tra l'iscrizione al Partito Nazionale Fascista e l'appartenenza alle logge massoniche.
} 
nota come massoneria sarebbe fondatrice e rifondatrice dei fasci e illustratrice della politica fascista ${ }^{21}$.

Per il lettore più attento, infine, ci preme evidenziare ancora un pregio di questo studio storico. Le analisi proposte da Padulo, per i diversi avvenimenti su cui ha concentrato la sua attenzione, sembrano svolgersi come indagini di polizia: iniziano con le domande su fatti e persone, espressamente scritte e non formulate solo nella mente dell'autore, per poi procedere alla loro validazione o falsificazione, con l'analisi incrociata del contenuto di documenti di diversa provenienza, fino alla deduzione ritenuta valida o più valida.

Vogliamo esprimere le ultime considerazione sugli strumenti dello storico che, però, avremmo preferito leggere in indici dedicati. La biografia utilizzata appare una solida base aggiornata con i principali testi pubblicati sull'argomento ${ }^{22}$ nonché con gli scritti di chi visse in prima persona quegli avvenimenti ${ }^{23}$, mentre riteniamo che meriti una nota particolare l'ampiezza della ricerca archivistica. Questa, effettuata in dodici archivi differenti setacciati da Padulo tra Roma, Genova, Salerno, Torino, Alessandria e Potenza, dimostra l'evidente formazione professionale dello storico autore, le sue capacità professionali e la conoscenza dell'argomento trattato.

\footnotetext{
${ }^{21}$ PADULO, Gerardo, L'ingrata progenie, cit., p. 158.

${ }^{22}$ Vedi nota 1.

${ }^{23}$ Tra gli altri, si segnalano, per lo specifico ambito massonico e per il riferimento dell'autore, gli scritti di: TORRIGIANI, Domizio, Ernesto Nathan. Parole dette in Palazzo Giustiniani, Roma, La Poligrafica Nazionale, 1922, ID., Massoneria e fascismo, Roma, La Poligrafica Nazionale, 1923, e quello di BANDINI, Gino, La massoneria per la guerra nazionale, Roma, Tipografia Ferraguti, 1924.
} 


\section{L'AUTORE}

Edoardo GRASSIA, diploma di laurea in Sociologia e laurea in Storia medievale, moderna e contemporanea presso l'Università La Sapienza di Roma, è dottorando di ricerca in Storia dell'Europa presso lo stesso ateneo.

URL: < http://www.studistorici.com/progett/autori/\#Grassia $>$ 